\title{
Robotic Approach to Cholecystectomy
}

\author{
Kaylene Barrera, Paul Chung and \\ Gainosuke Sugiyama \\ Additional information is available at the end of the chapter
}

http://dx.doi.org/10.5772/67517

\begin{abstract}
Cholecystectomy is one of the most commonly performed abdominal procedures with more than 600,000 performed annually in the United States. Laparoscopic cholecystectomy, first introduced in the 1980s, offered faster recovery time and a more cosmetic result making it the more favorable approach. In developed countries, up to $90 \%$ of cholecystectomies are done via laparoscopy. After the first robotic surgery platform was approved by the FDA in 2000, it provided surgeons with enhanced ergonomic capabilities and visualization and also offered possibility of telemedicine. The first series of robotic cholecystectomies soon followed in the last 15 years, and robotic cholecystectomy has become increasingly popular and has been established as a safe approach. The aims of this chapter are to address the history of robotic-assisted cholecystectomy, the technical aspects of multiport and single-port approaches, use of cholangiography, demonstration of safety and use in both community and academic settings.
\end{abstract}

Keywords: cholecystectomy, robotic surgery

\section{Introduction}

Today, in the United States, gallstone disease is one of the most common surgical diseases. An estimated 750,000 cholecystectomies are performed annually in the United States [1].

Management of gallstones has had a remarkable evolution in the last 20 years; however, mankind's journey in managing biliary disease has spanned more than 1600 years. The first description of gallstones was recorded by a Greek physician, Alexander Trallianus in the 500 AD. Early attempts to manage this disease included "cholagogues," which were medications thought to increase bile secretions and attempts to induce vomiting in an effort to dislodge the stones. Efforts to "dissolve" the stones were also unsuccessful. By the 1600s, 
experiments in dogs demonstrated that survival was possible after gallbladder removal. In 1743, Jean-Louis Petit performed the first "drainage" of the gallbladder through a percutaneous trocar [2-4]. It was not until 1867, that the first cholecystotomy was performed by Dr John Stough Bobbs. Bobbs opened the gallbladder, removed the gallstones within and then closed it [5]. This changed the philosophy of the management of gallbladder disease at that time. In 1882, Carl Langenbuch performed the first cholecystectomy in a patient concluding that the gallbladder should be removed "not because it contains stones, but because it forms them." Cholecystectomy then became a standard surgery for gallbladder disease [3].

The next landmark in gallbladder surgery was in 1985, when the first laparoscopic gallbladder surgery was performed by Dr Erich Mühe in Germany. Immediately he saw advantages over the traditional open approach with the immediate recovery stating "the approach was like magic." Unfortunately, he was met with much skepticism by colleagues who rejected this novel approach [6, 7]. It was not until laparoscopic cholecystectomy was performed in France that it began to spread globally. Dr Philippe Mouret of Lyon in France was a private surgeon who shared his practice with a gynecologist, who was performing laparoscopy. He too never published his achievement, stating "I did not see any chance for publishing in a surgical journal." Unlike Mühe, news of Mouret's success spread throughout France. Francois Dubois, a surgeon in Paris also performed a successful laparoscopic cholecystectomy and together with Jacques Perissat circulated news of this technique to the world [8, 9]. Laparoscopic cholecystectomy gradually became an attractive alternative to open cholecystectomy with its superior outcomes and is now the gold standard. The learning curve for laparoscopic surgery is long, requiring close training, with most complications occurring within the first 30 cases [10].

The next decade saw the introduction of single-site laparoscopic cholecystectomy, with the first reports published in 1995. This approach hoped to achieve even more enhanced cosmesis and decreased post-operative pain. Early versions of the technique utilized standard laparoscopic equipment via two $10 \mathrm{~mm}$ port incisions in the umbilicus. At the end of the procedure, the bridge of skin between the two incisions was cut to permit extraction of the gallbladder [11]. In 1997, this evolved to a single incision surgery technique where multiple ports could be placed through a single incision. In order to perform this type of surgery, surgeons use end articulating instruments and specialized ports. The most popular commercial ports are the TriPort ${ }^{\mathrm{TM}}$ (Olympus) and SILS port ${ }^{\mathrm{TM}}$ (Covidien) [12,13]. While the benefits of single-site surgery include cosmesis and are thought to reduce postoperative pain, concerns about the complications and technical aspect of this type of surgery remain a topic of controversy. Several large prospective randomized and case-matched cohorts have demonstrated no significant increases in complications, such as port site hernias or pain scores. While the procedure is slightly longer, with an average operative time of roughly 70 minutes compared to 55 minutes for multiport cholecystectomy, patients report greater satisfaction with the cosmetic result [13-16]. While the overall success and popularity of single-site surgery has been well described, the technical difficulty of the procedure remains a deterrent. Additional concerns about complications such as hernias, wound infection and increased pain have been demonstrated in prospective randomized controlled trials $[17,18]$. Furthermore single-site laparoscopic surgery is limited technically due to instrument collisions and the distance needed to travel from the umbilicus to the right upper quadrant. In order to operate effectively through a minimal access port, surgeons need to cross hand a difficult task to do laparoscopically (Figure 1). 
Robotic surgery has helped to overcome the challenges of conventional and single-site laparoscopy. In 2000, the FDA approved the first robotic surgery system. The first robotic cholecystectomy was performed on a human the following day [20]. Since then, the robotic-assisted platform has been applied to gynecologic, urologic, thoracic, colorectal and general surgery. Additionally, single-site cholecystectomy has also become increasingly popular.
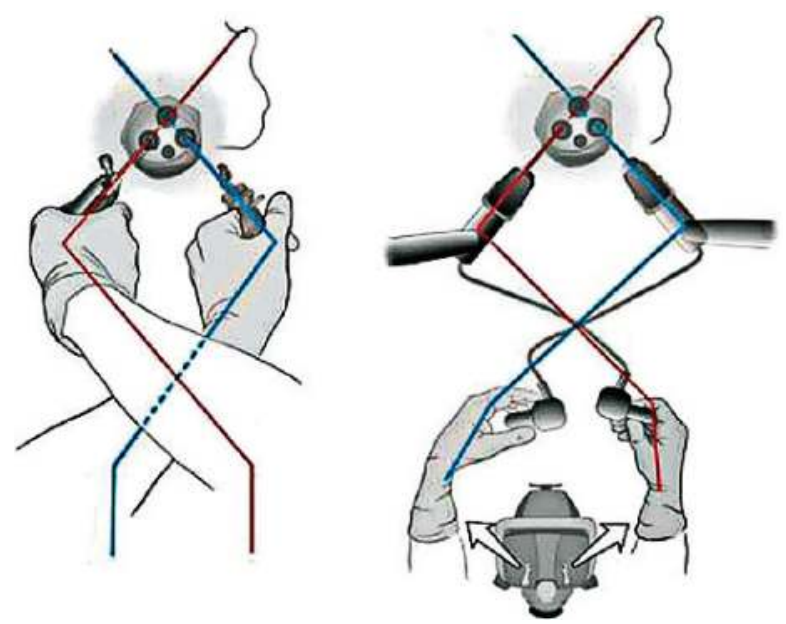

Figure 1. Single site surgery considerations: (L) Conventional laparoscopic instruments are challenging to use, and require the surgeon to cross instruments and arms in order to maintain maneuverability. (R) Robotic single site surgery these challenges into account and compensates for this, while allowing the surgeon to maintain their orientation. Ref. [19].

\section{General indications}

Indications for cholecystectomy include [21, 22]:

- Symptomatic cholelithiasis

- Biliary dyskinesia

- Acute cholecystitis

- Gallstone pancreatitis

Outpatient cholecystectomy can be performed in most patients; however, patients with acute cholecystitis or gallstone pancreatitis should be managed urgently.

Absolute contraindications include [23]:

- Inability to tolerate general anesthesia

- Suspicion of gallbladder cancer 


\section{Special considerations}

\subsection{Obesity}

Although once an exclusionary factor, robotic surgery is now performed regularly in obese patients, including single-site surgery. In a series of patients with BMI $\geq 30 \mathrm{mg} / \mathrm{m}^{2}$, the only significant difference in robotic cholecystectomy was a slightly longer operative time in obese patients (69.8 vs. 59.2 minutes, $p=0.001)$ [24].

\subsection{Pediatric patients}

Although we do not perform robotic cholecystectomy in pediatric patients in our practice, several studies have demonstrated that it can be performed safely. In a series of pediatric patients ranging 10-18 years, both multiport and single-site cholecystectomies were performed without complications [25]. Although laparoscopic cholecystectomy and robotic cholecystectomy have similar postoperative stays, concerns about the increased cost remain [26].

\subsection{Pregnancy}

Cholecystectomy in pregnant patients ranges from 1 case per 1100 to 10,000 live births [27]. According the Society of American Gastrointestinal and Endoscopic Surgeons (SAGES) guidelines, laparoscopic cholecystectomy is safe during all trimesters [28]. At the time of this writing, robotic-assisted cholecystectomy has not been reported in the literature. However, there are case reports of gynecologic and urologic procedures which include robotic partial nephrectomy, adrenalectomy and salpingo-oophorectomy being performed using the robotic platform. These procedures typically are performed during the second trimester [29-31]. The future of robotic cholecystectomy during pregnancy is yet to be determined.

\subsection{Anatomic variations and biliary imaging}

Biliary injuries occur in $0.2-0.8 \%$ of patients undergoing laparoscopic cholecystectomy [32, 33]. One of the suggested underlying causes is variant anatomy. Gallbladder and cystic duct anatomy can have many variations in all patients including: anomalous hepatic and cystic artery course (50\%), variations in insertion of the cystic duct and the common hepatic duct, duplicate gallbladder and cystic ducts $(0.03 \%)$, right segmental hepatic bile duct coursing close to cystic duct $(5 \%)$ or may have an absent cystic duct (rare) [34, 35]. The consequences of biliary injuries can be serious, requiring additional surgeries to reconstruct the biliary anatomy.

Measures to reduce the rate of biliary injuries include intraoperative imaging. For many years, cholangiography has been a mainstay of biliary imaging. Recently, especially in roboticassisted surgery, fluorescent imaging has become popular as it does not require cannulation of the cystic duct or additional radiation exposure.

The use of indocyanine green (ICG) to image the biliary tree was first described in 1992 [36]. ICG is a tricarbocyanine dye that is excreted into the bile. Peak concentration in the bile occurs 
at 120 minutes [37]. An intravenous dose of $2.5 \mathrm{mg}$ is given during administration of anesthesia or in the preoperative area. When illuminated with near infrared (NIR) light, ICG will emit light at a peak wavelength of $830 \mathrm{~nm}$. In order to view ICG in structures, the laparoscope must include a charge-coupled device (CCD) camera which can filter out wavelengths less than 810 nm [38]. In 2013, the Firefly ${ }^{\mathrm{TM}}$ Fluorescence Imaging Vision System was approved by the FDA for use with da Vinci ${ }^{\circledR}$ robotic platforms. Fluorescent image guidance can be used sporadically as verification or in real time. Use of indocyanine green has been repeatedly demonstrated as a safe technique in both laparoscopic and robotic-assisted cholecystectomy, allowing for visualization of the cystic duct, common bile duct and common hepatic duct in $94 \%$ or more of cases [39-41]. ICG can also be used to visualize the cystic artery if assessed within 45 seconds of an injection of ICG, but may lead to confusion between the vascular and biliary structures.

ICG is contraindicated in pregnancy, and in patients with allergies to iodine. Additionally, it is not an adequate tool to assess choledocholithiasis.

In our experience, ICG has been an important tool in cases of severe inflammation, helping to identify biliary structures in fibrinous areas and avoid conversion to open procedures (Figure 2).

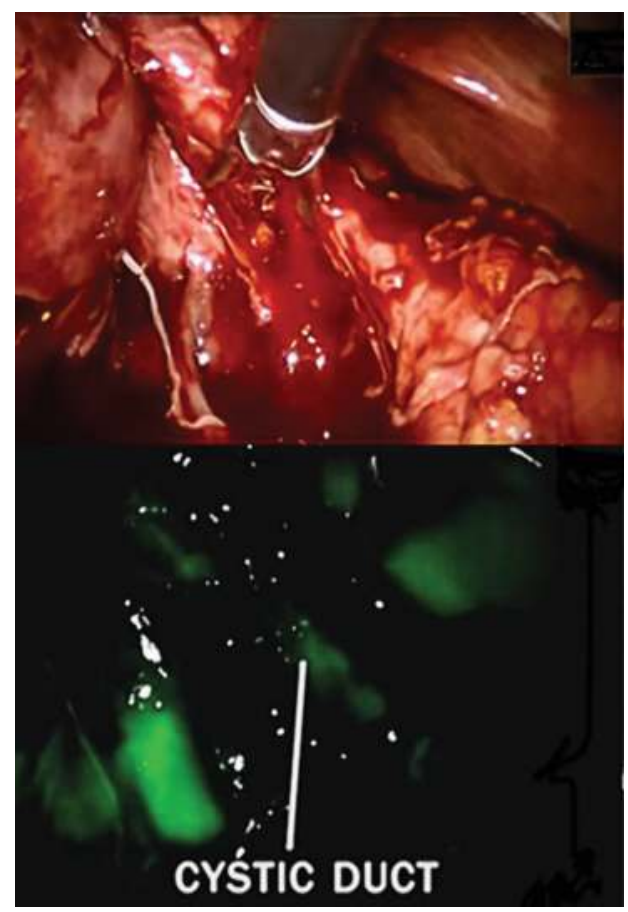

Figure 2. Top: cystic fundus and duct obscured by fibrinous tissue and adhesions. Bottom: cystic duct outlined by indocyanine green and near infrared imaging using the da Vinci Fluorescence Imaging Visual System. 


\section{Multiport robotic cholecystectomy}

\subsection{Instruments}

- Fundus grasper

- Monopolar cautery hook

- Hem-o-lok ${ }^{\circledR}$ clips and applier, or metal clips

- Curved scissors

- Maryland dissector

- Endocatch bag

- (Optional) AirSeal ${ }^{\circledR}$ system

\subsection{Patient preparation}

The patient is placed in the supine position with the arms tucked. General anesthesia is administered and the abdomen is then prepped and draped with the entire abdomen exposed.

If imaging of the biliary tree will be performed, indocyanine green is given intravenously ( 2.5 $\mathrm{mg})$.

\subsection{Port placement, docking}

In our operating room, the operating table is rotated $90^{\circ}$ after intubation. The robot is docked from the patient's right and anesthesia is at the head of the bed to the patient's left. A scrubbed assistant can stand to the patients left (Figure 3).

A $12 \mathrm{~mm}$ umbilical incision is made and a $12 \mathrm{~mm}$ robotic trocar is placed. After insufflation of the abdomen to $15 \mathrm{mmHg}$, an additional three ports are placed under direct visualization (Figure 4). In our practice, we use the AirSeal ${ }^{\circledast}$ (SurgiQuest, Inc., Milford, CT), to reduce smoke accumulation in the abdomen.

\subsection{Dissection}

After identification of the gallbladder, the fundus is retracted cephalad over the liver by the bedside assistant using a third robotic arm, or manually by a scrubbed assistant. In our practice, we do not require the third arm which reduces cost. The surgeon sits at the operating console. Adhesions are taken down using the Maryland dissector. Using an additional grasper, the gallbladder is retracted inferolaterally to expose the triangle of Calot.

The cystic duct and cystic artery are identified and further dissected using blunt techniques (Figure 5). NIR imaging can be used if the patient was given ICG. The critical view is then obtained after further dissection of the posterior peritoneum overlying the liver. The surgeon's 


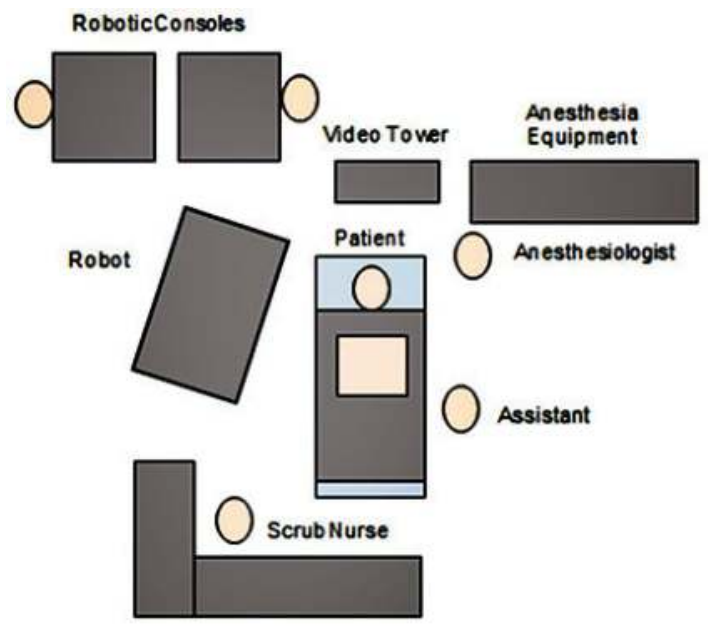

Instrument tables

Figure 3. Operating room setup.
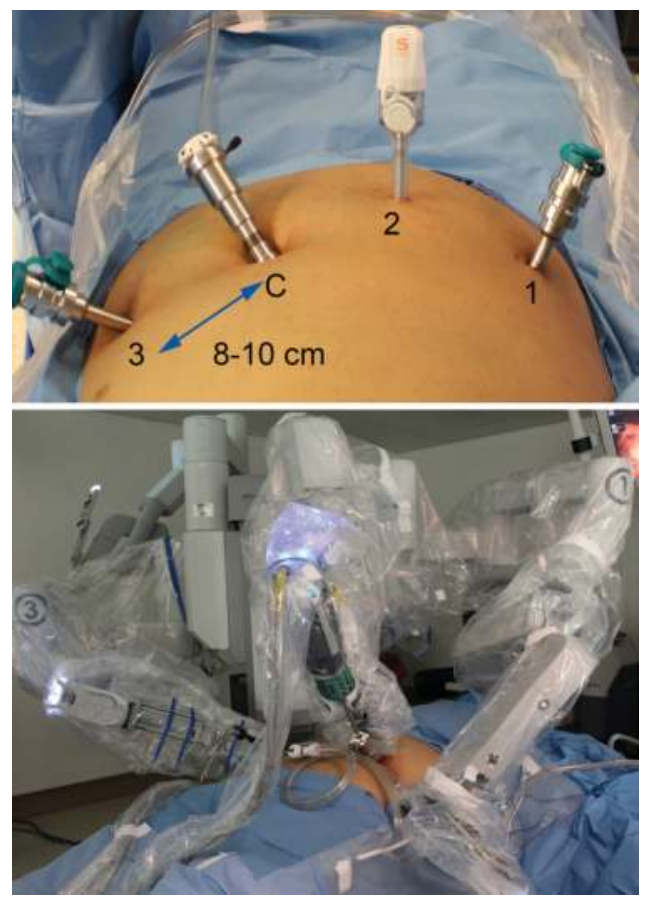

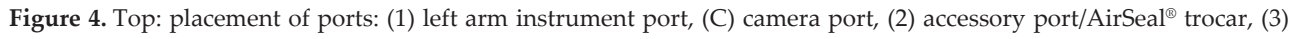
right arm instrument port. Bottom: docked robot. 


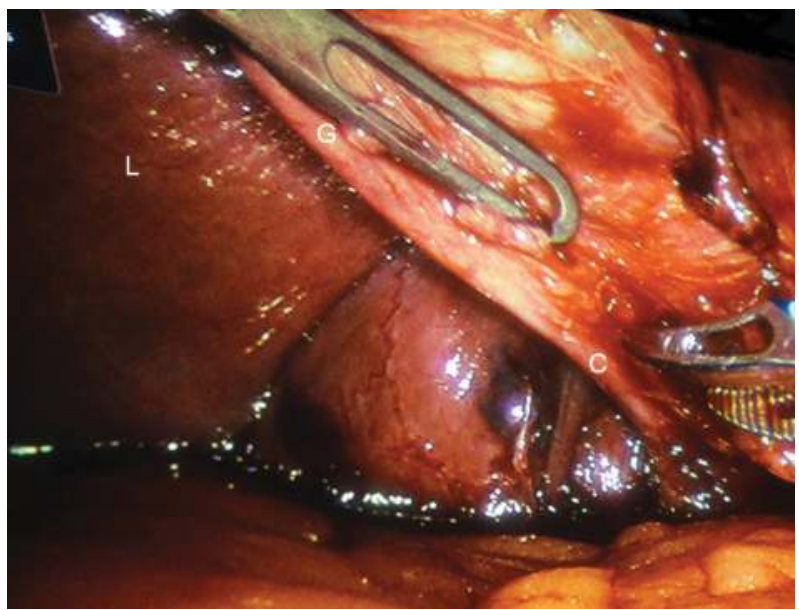

Figure 5. Blunt dissection of the cystic duct and artery. (L) liver, (C) cystic duct, (G) gallbladder.

right hand instrument is then exchanged for a Hem-o-lok ${ }^{\circledR}$ clip applier (or similar apparatus). The duct and artery are both clipped three times, with space to allow two clips to remain on proximal end of both the artery and duct.

The clip applier is then exchanged for a curved scissor to divide both the cystic artery and cystic duct. The gallbladder is then dissected from the liver surface using hook cautery. If a posterior branch of the cystic artery is encountered, this can also be ligated with Hem-o-lok ${ }^{\circledR}$ clips or cauterized. An additional option is bipolar cauterization of the cystic duct and arteries which has been demonstrated to be effective; however, the use is limited by additional expense [42].

Prior to disconnecting the gallbladder, it can be used to retract the liver to examine for bleeding. Additionally, the cystic artery and ductal stumps can be examined. After completion of the gallbladder resection, it can then be placed in an Endocatch bag and removed through the umbilical port.

The trocars are then removed under direct visualization. The fascia at the umbilicus is reapproximated with a figure of 8-0 vicryl stitch. The skin is reapproximated with interrupted 4-0 chromic sutures.

\section{Single-port robotic cholecystectomy}

In addition to the multiport technique, single port robotic cholecystectomy has become a popular modality made easier with the ergonomics afforded by the robotic platform. The design of the da Vinci ${ }^{\circledast}$ Single Site ${ }^{\circledR}$ platform minimizes instrument collisions by using curved trocars and flexible instruments. The first series on single-port robotic cholecystectomy were published in 2011. Subsequent studies, including randomized prospective trials demonstrated no difference in complications compared to conventional laparoscopic cholecystectomy but 
an increased preference by patients [43-45]. When compared to single-site laparoscopy, the robotic approach is associated with less pain [46].

\subsection{Instruments}

- da Vinci ${ }^{\circledR}$ Single Site ${ }^{\circledR}$ port

- Two 5 mm curved cannulae

- $5 \mathrm{~mm}$ semirigid instruments

○ Maryland dissector

○ Monopolar cautery hook

○ Hem-o-lok ${ }^{\circledast}$ clips and applier, or metal clips

- Curved scissors

- Endocatch bag

\subsection{Patient preparation}

Patient positioning is similar to multiport robotic-assisted cholecystectomy. The patient is in the supine position and arms are tucked. General anesthesia is administered and the abdomen is prepped and draped in a similar fashion. If imaging of the biliary tree will be performed, indocyanine green is given at least 45 minutes prior to visualization.

\subsection{Port placement, docking}

As with multiport cholecystectomy, the operating table is rotated $90^{\circ}$ after intubation. The robot is docked from the patient's right and anesthesia is at the head of the bed to the patient's left. A scrubbed assistant stands to the patients left. The patient is placed in reverse Trendelenburg position to allow the intestines to fall away from the liver and gallbladder bed.

A $2.5 \mathrm{~cm}$ vertical umbilical incision is made and extended to the fascia. A finger sweep is performed to clear the area of adhesions and bowel. A multiport da Vinci ${ }^{\circledR}$ Single-Site $^{\circledR}$ port (Intuitive Surgical Inc., Sunnyvale, CA, USA) is then placed inside. Wetting the port and or using an $S$ retractor can help facilitate placement. In our practice, we secure the port in place to minimize movement with 2-0 nylon sutured from the edges of the port superficially to the skin at four points.

The port used with the da Vinci Si Surgical System includes five lumens including an insufflation adapter, accessory port, two-curved cannulae ports and a camera port (Figure 6). After insufflation, the curved cannulae are placed until the first black line is visible within the abdomen. The right-sided cannula is operated by the surgeon's left hand, and the left-sided cannula is operated by the surgeon's right hand. Care must be taken to not create a false tract within the single-site port by forcing entry of the cannula. 


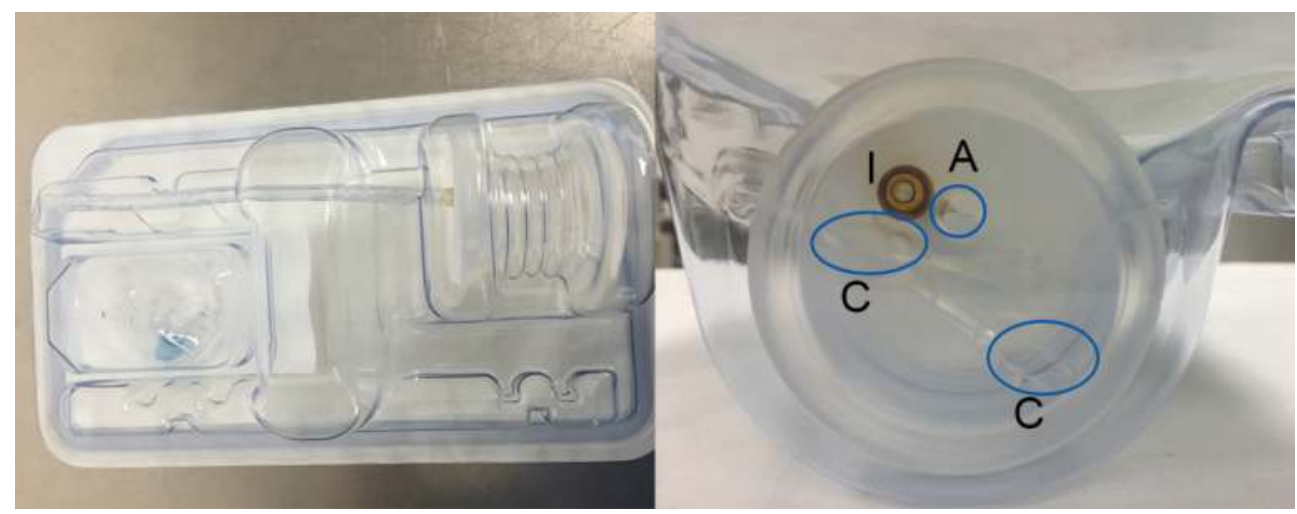

Figure 6. da Vinci ${ }^{\circledast}$ Single Site ${ }^{\circledast}$ multiport system. Left: side view, Right: intra-abdominal side/port sites. (C) curved cannulae, (I) insufflation port, (A) accessory port.

\subsection{Dissection}

After identification of the gallbladder, a standard laparoscopic grasper is placed through the accessory port. This must also be accomplished carefully so as not to create a false tract within the single-site port. The bedside assistant retracts the gallbladder fundus cephalad and over the liver. The surgeon sits at the operating console. Adhesions are taken down using the Maryland dissector. Using an additional grasper, the gallbladder is retracted inferolaterally.

The cystic duct and artery are dissected bluntly. If needed, near infrared (NIR) imaging can be used to visualize the biliary anatomy 45 minutes after administration. As with the multiport technique, dissection continues until the critical view is obtained. The surgeon's right hand instrument is then exchanged for a Hem-o-lok clip applier (or equivalent). Three clips are placed on each duct and artery and transected above the first two clips with robotic Endoshears.

The gallbladder is then dissected from the liver bed using hook cautery, using the same principles described in multiport robotic cholecystectomy. The abdomen is inspected for hemostasis and the gallbladder placed in an Endocatch bag.

The single-site port is then released from the stay sutures and removed. The fascia at the umbilicus is reapproximated with a running 0 -vicryl. The skin is reapproximated with interrupted 4-0 chromic.

\section{Conversion to open}

Indications to convert to an open procedure include adhesions, suspected biliary duct injury, bowel injury and hemorrhage.

Robotic surgery and utilization of ICG may lead to a reduction in rates of conversion to open surgery $[47,48]$. In a large series of laparoscopic cholecystectomy, the rate of conversion is reported 
as $2.6-3 \%$, with adhesions being the most common reason for conversion [49, 50]. When compared to robotic surgery in a recent meta-analysis, conversion rate ranged as high as $15.7 \%$ for laparoscopic compared to $1.9 \%$ in robotic surgery, but did not reach statistical significance [51].

\section{Cost}

A significant concern about the utilization of robotic surgery is the associated cost which has been a topic of debate. Analyses of the outpatient costs of robotic-assisted cholecystectomy show higher total charges and costs when compared to laparoscopic surgery. However, proponents cite the numerous benefits of robotic surgery including enhanced surgeon ergonomics and the potential for building skills to perform more complex operations robotically [48, 52]. In pediatric patients hospitalization cost for robotic compared to non-robotic averaged $\$ 11,000$ vs. $\$ 7000$ [26].

The increased cost in robotic surgery, however, may be a related to it being a relatively new technology with limited competition. In hospitals with an established infrastructure for robotic surgery, there is potential for cost efficacy. In a review from one institution, overall savings from supplies and instruments and shortened operating room times resulted in robotic single-site laparoscopic cholecystectomy being more cost effective than laparoscopic cholecystectomy [53]. When comparing cost margin only at a private community hospital, there was no difference in cost between robotic and laparoscopic cholecystectomy [54].

A similar concern regarding cost existed when laparoscopic cholecystectomy was first introduced. Although laparoscopic surgery had increased costs, the savings resulted from decreased hospital stays [55]. Today, cholecystectomy is performed routinely as an outpatient procedure, and those that are hospitalized are able to be discharged after 1 day. A possible area where robotic surgery can present a cost benefit is in the use of ICG vs. cholangiography and reduction in biliary injuries and subsequent surgeries and hospitalization.

\section{Outcomes}

\subsection{Biliary injury}

With the integration of the Fluorescence Visual Imaging System, biliary imaging is readily available following the injection of ICG. In a comparison to laparoscopic cholecystectomy, robotic cholecystectomies were found to have less open conversion, less major biliary injuries and increased identification of biliary anomalies [47].

\subsection{Hernia}

Port site hernias remain a concern of single-site surgeries. In the laparoscopic literature, reported rates range between 2.9 and $8.4 \%[17,56]$. Data from robotic single-site surgery are limited to smaller case series. In a retrospective series of 27 patients, $5(19 \%)$ trocar-site hernias 
were reported [57]. In a retrospective study of 112 obese patients, there was only 1 incisional hernia $(0.9 \%)$ [24]. Further long-term studies are needed to further describe the scope of this complication.

\subsection{Postoperative pain}

Robotic surgery is theorized to cause less post-operative pain due to less torque applied to the incision sites. A retrospective study comparing single-site robotic cholecystectomy and single incision laparoscopic cholecystectomy demonstrated lower post-operative pain scores in the robotic group [46]. Conversely, in a randomized double-blind trial comparing singlesite robotic cholecystectomy to laparoscopic cholecystectomy, there were no significant differences between the two groups [58].

\section{Robotic cholecystectomy and surgical education}

Robotic cholecystectomies are currently performed in a broad range of hospital settings from community to academic teaching institutions. Trainee involvement does not affect outcomes $[54,59]$. In our institution, resident trainees develop robotic skills on a simulator, and gradually acquire the skills required to perform the dissection. We anticipate that robotic skills will be an essential part of the surgeon's toolkit. Further evaluation of the learning curve of robotic surgery in graduate medical education is warranted.

\section{Future directions}

In addition the da Vinci ${ }^{\circledR}$ platform, several other new systems are being introduced.

The Revo- $\mathrm{I}^{\circledR}$ Model MSR-5000 is currently undergoing animal study in robotic cholecystectomy. Similar to the da Vinci ${ }^{\circledR}$ system, it offers 3D visualization, tremor filtration and 7 degrees of freedom. However, the current machine is limited to monopolar and bipolar energy sources [60].

One criticism of robotic surgery compared to laparoscopic surgery is the absence of haptic feedback. The Telelap ALF- ${ }^{\circledR}$ provides haptic feedback, and the developers of the Revo-i ${ }^{\circledR}$ are reportedly developing a haptic feedback component [60]. The Telelap ALF- ${ }^{\circledR}$ has been used to date in gynecologic and urological procedures.

\section{Conclusions}

Robotic cholecystectomy offers a safe modality to continue treating biliary disease. The continued study of this technique will identify potential safety and cost benefits. Continued development of new robotic technologies may further diversify the field and curb economic concerns. 


\section{Author details}

Kaylene Barrera, Paul Chung and Gainosuke Sugiyama*

*Address all correspondence to: gainosuke.sugiyama@downstate.edu

State University of New York, Downstate Medical Center, Brooklyn, New York, USA

\section{References}

[1] Flum, D.R., et al., Intraoperative cholangiography and risk of common bile duct injury during cholecystectomy. JAMA, 2003. 289(13): pp. 1639-44.

[2] Bielefeldt, K., Black bile of melancholy or gallstones of biliary colics: historical perspectives on cholelithiasis. Dig Dis Sci, 2014. 59(11): pp. 2623-34.

[3] Norton, J.A., Surgery Basic Science and Clinical Evidence. 2008, Springer: New York, NY. p. 1 online resource (xliv, 2442 pages) illustrations, portraits.

[4] De, U., Evolution of cholecystectomy: a tribute to Carl August Langenbch. Indian J Surg, 2004. 66(2): pp. 97-100.

[5] Sparkman, R.S., Bobbs centennial: the first cholecystotomy. Surgery, 1967. 61(6): pp. 965-71.

[6] Litynski, G.S., Erich Muhe and the rejection of laparoscopic cholecystectomy (1985): a surgeon ahead of his time. JSLS, 1998. 2(4): pp. 341-6.

[7] Reynolds, W., Jr., The first laparoscopic cholecystectomy. JSLS, 2001. 5(1): pp. 89-94.

[8] Litynski, G.S., Profiles in laparoscopy: Mouret, Dubois, and Perissat: the laparoscopic breakthrough in Europe (1987-1988). JSLS, 1999. 3(2): pp. 163-7.

[9] Dubois, F., et al., Coelioscopic cholecystectomy. Preliminary report of 36 cases. Ann Surg, 1990. 211(1): pp. 60-2.

[10] Cagir, B., et al., The learning curve for laparoscopic cholecystectomy. J Laparoendosc Surg, 1994. 4(6): pp. 419-27.

[11] Navarra, G., et al., One-wound laparoscopic cholecystectomy. Br J Surg, 1997. 84(5): p. 695.

[12] Romanelli, J.R., Mark, L., Omotosho, P.A., Single port laparoscopic cholecystectomy with the TriPort system: a case report. Surg Innov, 2008. 15(3): pp. 223-8.

[13] Ross, S., et al., Consensus statement of the consortium for LESS cholecystectomy. Surg Endosc, 2012. 26(10): pp. 2711-6.

[14] Arezzo, A., Passera, R., Bullano, A. etal.Surg Endosc (2016). doi:10.1007/s00464-016-5298-7 
[15] Wong, J.S., et al., Comparison of postoperative pain between single-incision laparoscopic cholecystectomy and conventional laparoscopic cholecystectomy: prospective case-control study. Surg Laparosc Endosc Percutan Tech, 2012. 22(1): pp. 25-8.

[16] Cheng, Y., et al., Laparoendoscopic single-site cholecystectomy vs three-port laparoscopic cholecystectomy: a large-scale retrospective study. World J Gastroenterol, 2013. 19(26): pp. 4209-13.

[17] Marks, J.M., et al., Single-incision laparoscopic cholecystectomy is associated with improved cosmesis scoring at the cost of significantly higher hernia rates: 1-year results of a prospective randomized, multicenter, single-blinded trial of traditional multiport laparoscopic cholecystectomy vs single-incision laparoscopic cholecystectomy. J Am Coll Surg, 2013. 216(6): pp. 1037-47; discussion 1047-8.

[18] Phillips, M.S., et al., Intermediate results of a prospective randomized controlled trial of traditional four-port laparoscopic cholecystectomy versus single-incision laparoscopic cholecystectomy. Surg Endosc, 2012. 26(5): pp. 1296-303.

[19] Dhumane, P.W., Diana, M., Leroy, J., Marescaux, J., Minimally invasive single-site surgery for the digestive system: a technological review. J Min Acc Surg, 2011. 7(1): 40-51. DOI: $10.4103 / 0972-9941.72381$

[20] Eisner, R., FDA OKs first robotic surgical device. ABC News, 2000 [cited 2016 12/23/2016]; Available from: http://abcnews.go.com/Health/story?id=118152\&page=1.

[21] NIH releases consensus statement on gallstones, bile duct stones and laparoscopic cholecystectomy. Am Fam Physician, 1992. 46(5): pp. 1571-4.

[22] Bellows, C.F., Berger, D.H., Crass, R.A., Management of gallstones. Am Fam Physician, 2005. 72(4): pp. 637-42.

[23] Nguyen, K.T., Birkmeyer, J.D., Chapter 11 Laparoscopic Cholecystectomy, in Current Procedures: Surgery, R.M. Minter, G.M. Doherty, Editors. 2010, The McGraw-Hill Companies: New York, NY.

[24] Svoboda, S., et al., Robotic single-site cholecystectomy in the obese: outcomes from a single institution. Surg Obes Relat Dis, 2015. 11(4): pp. 882-5.

[25] Ahn, N., et al., Robotic single- and multisite cholecystectomy in children. J Laparoendosc Adv Surg Tech A, 2015. 25(12): pp. 1033-5.

[26] Mahida, J.B., et al., Utilization and costs associated with robotic surgery in children. J Surg Res, 2015. 199(1): pp. 169-76.

[27] Ramin, K.D., Ramsey, P.S., Disease of the gallbladder and pancreas in pregnancy. Obstet Gynecol Clin North Am, 2001. 28(3): pp. 571-80.

[28] Pearl, J., et al., Guidelines for diagnosis, treatment, and use of laparoscopy for surgical problems during pregnancy. Surg Endosc, 2011. 25(11): pp. 3479-92.

[29] Ramirez, D., et al., Robotic partial nephrectomy during pregnancy: case report and special considerations. Urology, 2016. 92: pp. 1-5. 
[30] Chen, C.H., et al., Management of ovarian cancer in 14th gestational week of pregnancy by robotic approach with preservation of the fetus. Gynecol Obstet Invest, 2015. 80(2): pp. 139-44.

[31] Podolsky, E.R., et al., Robotic resection of pheochromocytoma in the second trimester of pregnancy. JSLS, 2010. 14(2): pp. 303-8.

[32] Giger, U., et al., Bile duct injury and use of cholangiography during laparoscopic cholecystectomy. Br J Surg, 2011. 98(3): pp. 391-6.

[33] Shamiyeh, A., Wayand, W., Laparoscopic cholecystectomy: early and late complications and their treatment. Langenbecks Arch Surg, 2004. 389(3): pp. 164-71.

[34] Pham, T.H., Hunter, J.G., Gallbladder and the Extrahepatic Biliary System, in Schwartz's Principles of Surgery, 10th ed., F.C. Brunicardi, et al., Editors. 2014, McGraw-Hill Education: New York, NY.

[35] Turner, M.A., Fulcher, A.S., The cystic duct: normal anatomy and disease processes. Radiographics, 2001. 21(1): pp. 3-22; questionnaire 288-94.

[36] Araki, K., et al., Indocyanine green staining for visualization of the biliary system during laparoscopic cholecystectomy. Endoscopy, 1992. 24(9): p. 803.

[37] Cherrick, G.R., et al., Indocyanine green: observations on its physical properties, plasma decay, and hepatic extraction. J Clin Invest, 1960. 39: pp. 592-600.

[38] Ishizawa, T., Bandai, Y., Kokudo, N., Fluorescent cholangiography using indocyanine green for laparoscopic cholecystectomy: an initial experience. Arch Surg, 2009. 144(4): pp. 381-2.

[39] Daskalaki, D., et al., Indocyanine green (ICG) fluorescent cholangiography during robotic cholecystectomy: results of 184 consecutive cases in a single institution. Surg Innov, 2014. 21(6): pp. 615-21.

[40] Spinoglio, G., et al., Real-time near-infrared (NIR) fluorescent cholangiography in single-site robotic cholecystectomy (SSRC): a single-institutional prospective study. Surg Endosc, 2013. 27(6): pp. 2156-62.

[41] Ishizawa, T., et al., Fluorescent cholangiography illuminating the biliary tree during laparoscopic cholecystectomy. Br J Surg, 2010. 97(9): pp. 1369-77.

[42] Schulze, S., et al., Cystic duct closure by sealing with bipolar electrocoagulation. JSLS, 2010. 14(1): pp. 20-2.

[43] Kudsi, O.Y., Castellanos, A., Kaza, S. et al. Surg Endosc (2016). doi:10.1007/s00464016-5353-4

[44] Kroh, M., et al., First human surgery with a novel single-port robotic system: cholecystectomy using the da Vinci Single-Site platform. Surg Endosc, 2011. 25(11): pp. 3566-73.

[45] Wren, S.M., Curet, M.J., Single-port robotic cholecystectomy: results from a first human use clinical study of the new da Vinci single-site surgical platform. Arch Surg, 2011. 146(10): pp. 1122-7. 
[46] Su, W.L., et al., Comparison study of clinical outcomes between single-site robotic cholecystectomy and single incision laparoscopic cholecystectomy. Asian J Surg, 2016. http:// dx.doi.org/10.1016/j.asjsur.2016.03.005

[47] Gangemi, A., Danilkowicz, R., Elli, F.E. et al. J Robotic Surg (2017) 11: 77. doi:10.1007/ s11701-016-0624-6

[48] Strosberg, D.S., Nguyen, M.C., Muscarella, P. et al. Surg Endosc (2017) 31: 1436. doi:10. 1007/s00464-016-5134-0

[49] Genc, V., et al., What necessitates the conversion to open cholecystectomy? A retrospective analysis of 5164 consecutive laparoscopic operations. Clinics (Sao Paulo), 2011. 66(3): pp. 417-20.

[50] Le, V.H., Smith, D.E., Johnson, B.L., Conversion of laparoscopic to open cholecystectomy in the current era of laparoscopic surgery. Am Surg, 2012. 78(12): pp. 1392-5.

[51] Huang, Y., et al., Robotic cholecystectomy versus conventional laparoscopic cholecystectomy:a meta-analysis. Surgery, 2016. 161(3) 628-636.

[52] Newman, R.M., et al., Surgical value of elective minimally invasive gallbladder removal: a cost analysis of traditional 4-port vs single-incision and robotically assisted cholecystectomy. J Am Coll Surg, 2016. 222(3): pp. 303-8.

[53] Bedeir, K., Mann, A., Youssef, Y., Robotic single-site versus laparoscopic cholecystectomy: which is cheaper? A cost report and analysis. Surg Endosc, 2016. 30(1): pp. 267-72.

[54] Hawasli, A., et al., The impact of robotic cholecystectomy on private practice in a community teaching hospital. Am J Surg, 2016. 211(3): pp. 610-4.

[55] Schirmer, B.D., Dix, J., Cost effectiveness of laparoscopic cholecystectomy. J Laparoendosc Surg, 1992. 2(4): pp. 145-50.

[56] Agaba, E.A., et al., Incidence of port-site incisional hernia after single-incision laparoscopic surgery. JSLS, 2014. 18(2): pp. 204-10.

[57] van der Linden, Y.T., et al., Robotic single-port laparoscopic cholecystectomy is safe but faces technical challenges. J Laparoendosc Adv Surg Tech A, 2016. 26(11): pp. 857-61.

[58] Pietrabissa, A., et al., Short-term outcomes of single-site robotic cholecystectomy versus four-port laparoscopic cholecystectomy: a prospective, randomized, double-blind trial. Surg Endosc, 2016. 30(7): pp. 3089-97.

[59] Chung, P.J., et al., Single-site robotic cholecystectomy at an inner-city academic center. JSLS, 2015. 19(3).

[60] Lim, J.H., Lee, W.J., Park, D.W. et al. Surg Endosc (2016). doi:10.1007/s00464-016-5357-0 